\title{
Special Issue on Al-Driven Smart Networking and Communication for Personal Internet of Things: Part II
}

\author{
Yong $\mathrm{Jin}^{1} \cdot \mathrm{Honghao} \mathrm{GaO}^{2} \cdot \mathrm{TaO}^{\mathrm{Hu}^{3}} \cdot$ Xinrong $\mathrm{Li}^{4}$
}

Published online: 13 April 2020

○) Springer Science+Business Media, LLC, part of Springer Nature 2020

With the rapid development of personal computing technology and intelligent devices, the Internet of Things (IoT) industry has reached a new level for consumers. Compared with the conventional IoT, Personal IoT integrates various ubiquitous networking, communication, sensing, and control capabilities, as well as intelligent analysis and decision technologies, into all aspects of personal daily life process. Nowadays, artificial intelligence (AI) facilitates ubiquitous smart networking and communication, application analysis and prediction, and thus to make an optimal decision. The basic idea of Personal IoT is to leverage a multitude of collaborative ubiquitous personal smart devices and near-user infrastructures to carry out a substantial amount of personal data retrieval, recognition, compression, and communication tasks with low latency, energy efficiency, and many other desirable features.

Despite tremendous benefits and opportunities that the AI offers, there are still many technical challenges that need to be addressed by the research and development communities to realize the full potentials of the Personal IoT. The goal of this special issue is to attract high quality contributions that collectively provide a comprehensive coverage of recently developments related to the AI-driven Personal IOT technology and applications.

Yong Jin

jinyong@cslg.edu.cn

Honghao Gao

honghaogao@hotmail.com

$\mathrm{Tao} \mathrm{Hu}$

hutao.ohio@gmail.com

Xinrong Li

Xinrong.Li@unt.edu

Changshu Institute of Technology, Changshu, China

2 Shanghai University, Shanghai, China

3 Kent State University, Kent, OH, USA

4 University of North Texas, Denton, TX, USA
The papers published in this special issue are accepted through an open call for papers. The submitted manuscripts were reviewed by experts from both academia and industry. This special issue will be published by International Journal of Wireless Information Networks in two parts. This is the second part of the special issue includes twelve papers.

The paper "A Network Attack Detection Method Using SDA and Deep Neural Network Based on Internet of Things" by Jingwei $L i$ et al. proposed this paper proposes a network attack detection method using deep neural networks in the Internet of Things based on a comprehensive analysis of network attacks and deep neural networks. The paper "Data Acquisition and Transmission Scheme for Large Projects Based on LoRa Internet of Things Using Improved Linear Integer Programming Model" by Jun Hu et al. designed an environmental monitoring system based on LoRa wireless sensor network, with the characteristics of long transmission distance and low power consumption on LoRa. The paper "A Massive Multi-Modal Perception Data Classification Method Using Deep Learning Based on Internet of Things" by Linli Jiang et al. proposed the heterogeneous multi-source multi-modal perception data classification and detection to ensure the quality of data and select some data sources for data transmission in order to save network resources. The paper "A Sink Node Trusted Access Authentication Protocol for Mobile Wireless Sensor Network Using Block Cipher Algorithm Based on IoT" by Qian Wang et al. proposed a sink node trusted access authentication protocol using packet cipher algorithm for mobile WSN. The paper "Long Link Wireless Sensor Routing Optimization Based on Improved Adaptive Ant Colony Algorithm" by Qian Zhou et al. studied the characteristics of wireless sensor network and the challenges of wireless sensor network routing protocol. The paper "A Novel Important Node Discovery Algorithm Based on Local Community Aggregation and Recognition in Complex Networks" by Qu-zhi Huang, proposed a novel important node discovery algorithm based on local community aggregation and recognition in complex Networks. 
The paper "Distance Mapping Algorithm for Sensor Node Localization in WSNs" by Rong Tan et al. proposed a node localization algorithm for improving the localization of sensing nodes in the application of wireless sensor networks. The paper "Compression Sensing Signal Detection Algorithm Based on Orthogonal Matching Pursuit" by Shen Jian et al. proposed a compression sensing signal detection algorithm based on orthogonal matching pursuit. The paper "Energy-Saving Optimization and Matlab Simulation of Wireless Networks Based on Clustered Multi-hop Routing Algorithm" by Shun Liu, proposed a real-time routing protocol by combining the location information and clustering technology to reduce the energy consumption of the wireless sensor network. The paper "Tyson Polygon Construction Based on Spatio-temporal Data Network" by Xiaoming $B i$, studied the problem of data management and topology transformation of spatio-temporal networks in the face of complex data processing. The paper "A Hadoop Processing Method for Massive Sensor Network Data Based on Internet of Things" by Yanxin Zhang, proposed a mass sensing information processing scheme based on IoT service platform based on the analysis of the architecture of the Internet of Things service platform and the key technologies of cloud computing. The paper "Coverage Optimization and Simulation of Wireless Sensor Networks Based on Particle Swarm Optimization" by Ye Zhang, studied the coverage optimization of wireless sensor networks based on an improved particle swarm optimization.

We would like to thank all reviewers who have helped in the paper review process, and the authors for their contribution and efforts to complete the papers with a very high quality. Finally, we express our gratitude to Dr. Kaveh Pahlavan for initiating this special issue and inviting us to undertake this rewarding activity.

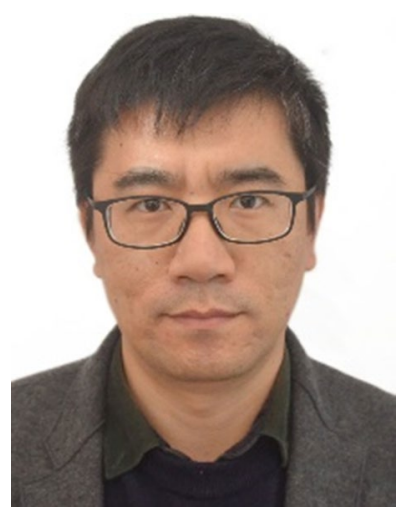

Yong Jin is currently an associate professor in the school of computer science and engineering at Changshu Institute of Technology. His recent research has been focused on wireless sensor networks, fog computing, wireless charging system, Industrial Internet of Things, deep transfer learning, etc.

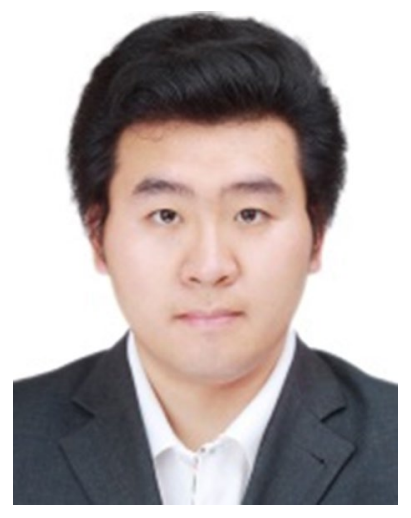

Honghao Gao received the Ph.D. degree in Computer Science and started his academic career at Shanghai University in 2012. He is an IET Fellow, BCS Fellow, EAI Fellow, IEEE Senior Member, CCF Senior Member, and CAAI Senior Member. Prof. Gao is currently a Distinguished Professor with the Key Laboratory of Complex Systems Modeling and Simulation, Ministry of Education, China. His research interests include service computing, model checking-based software verification, and sensors data

application.

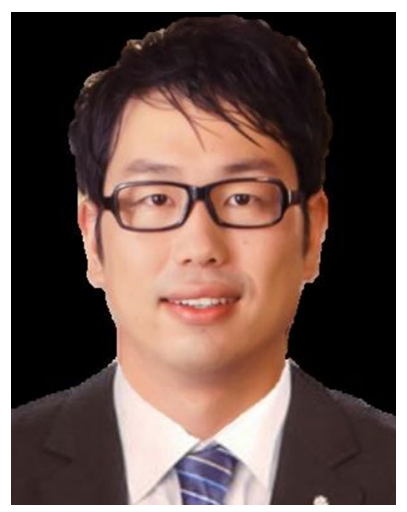

Tao Hu received his $\mathrm{Ph} . \mathrm{D}$. at Wuhan University, China in 2015 , and he was a post-doctoral fellow at School of Information in Kent State University from 2016 to 2017. Currently, he is the joint post-doctoral research fellow of University of Michigan and Kent State University. Dr. $\mathrm{Hu}$ is working on the spatial intelligence platform development. Dr. Hu's research interests include human behavior analysis, high performance computing, spatial-temporal big data analysis and visualization.

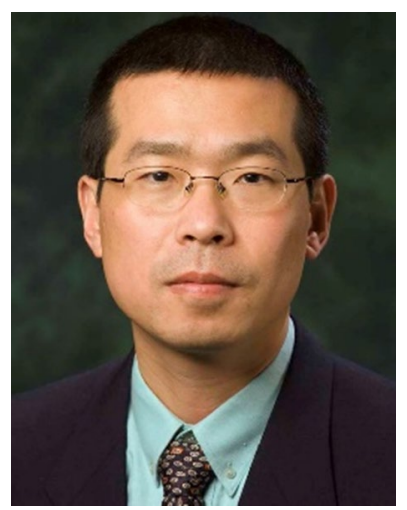

Xinrong $\mathrm{Li}$ received $\mathrm{BE}$ degree from the University of Science and Technology of China, Hefei, China, in 1995, ME degree from the National University of Singapore in 1999, and Ph.D. degree from Worcester Polytechnic Institute (WPI), Worcester, MA, in 2003, all in Electrical Engineering. From 2003 to 2004, he was a Post-doc Research Fellow at the Center for Wireless Information Network Studies, WPI. He joined the Department of Electrical Engineering, University of North Texas, Denton, Texas, as an Assistant Professor in 2004, and then he was tenured and promoted to Associate Professor in 2010. His recent research has been focused on statistical signal processing, machine learning, geolocation, and wireless sensor network systems. 